\title{
PRÁCTICAS EMERGENTES, ESPACIO CURATORIAL Y EMPLAZAMIENTO SUR
}

\author{
EMERGING PRACTICES, CURATORIAL FIELD AND SOUTHERN LOCATION
}

EVA NATALIA FERNÁNDEZ

Universidad Autónoma de Querétaro, México

http://orcid.org/0000-0003-4437-8104

evafernandezfotografia@gmail.com
Recepción: 21 de julio de 2020

Aprobación: 26 de octubre de 2020
RESUMEN

El siguiente texto ofrece una reflexión en torno al ejercicio de las "prácticas curatoriales" aplicadas al territorio del arte y al espacio expositivo, con la intención de caracterizar los derroteros que éstas tienen, distinguiendo dos modos del saber hacer curatorial: el primero, vinculado a una práctica hegemónica, avalada y sostenida por el discurso oficial del campo curatorial institucionalizado; y el segundo, el de una práctica disidente, establecida por su oposición a la narrativa canónica y enunciada desde los márgenes. Esta clasificación teórica es una propuesta del historiador del arte y curador Marcelo Pacheco, y en este trabajo se añade a esa coyuntura la problemática de pensar las prácticas curatoriales desde un emplazamiento específico -el espacio geopolítico Sur - con el objetivo de explorar las potencialidades de una práctica curatorial que trascienda lo local.

Palabras clave: campo curatorial, discursos, espacio geopolítico, prácticas, Sur.
ABSTRACT

This article offers a reflection on the exercise of curatorial practices applied to the territory of art and the exhibition space, with the intention of characterizing the courses they have, distinguishing two way of curatorial know-how. The first, linked to a hegemonic practice, endorsed and support by the official discourse of the institutionalized curatorial field, and, the second, that of a dissident practice, established by its opposition to the canonical narrative and enunciated from the border. This theoretical classification is a proposal of the art historian and curator Marcelo Pacheco, and in this work is added to this joint the problem of thinking about curatorial practices from a specific location -the southern geopolitical space- whit the aim of exploring the potential of a curatorial practice that transcends the local.

Key words: curatorial field, discourses, geopolitical site, practices, South. 
ARTí́CULO

EVA NATALIA FERNÁNDEZ

Prácticas emergentes, espacio

curatorial y emplazamiento Sur
INTRODUCCIÓN

Al inició de la redacción de este artículo, fue difícil decidir si empezar por el "discurso" o por las "prácticas", algo así como la fábula del huevo y la gallina. Aunque el objeto del análisis era uno y muy claro, la curaduría, la comprensión del fenómeno curatorial -con sus múltiples variables- no parecía ceñirse a un simple marco de interpretación o a la posibilidad de arrojar un único resultado.

En esa búsqueda por discernir, una opción fue concebir al discurso curatorial previo a la práctica y con una función clave: "comunicar algo". La relevancia que adquiere el sentido en el discurso curatorial, incluso desde una dimensión metafórica, parece sostener una estructura ideológica y política que, como recurso ostensible de utilizarse, se sintetiza en el gusto del curador y su posición institucional. A simple vista, podría pensarse que esta figura - la del curador - no hace más que mediar entre el espacio expositivo y el público, sin embargo, su trabajo va más allá de una escueta intervención, negociación y gestión de la diferencia.

La otra opción fue suponer que los discursos emergen luego de las prácticas - museales, curatoriales, artísticas o culturales-y que son ellas -las prácticas - las que posibilitan "[...] circular significados e interpretaciones por senderos múltiples, sin vigilancia" (Pacheco, 2001). Finalmente, la conclusión fue que no era una ni otra, sino ambas.

Por eso, el texto del curador y crítico mexicano Cuauhtémoc Medina, "Curando desde el Sur: una comedia en cuatro actos", da algunas pistas para elaborar una respuesta posible a la disyuntiva antes planteada, allí afirma que "[...] el mundo y las prácticas artísticas, institucionales, redes y teorías están profundamente entrelazadas e implicadas entre sí" (Medina, 2015: 114) De esta manera, la confusión de pensarlas en un orden, pone de manifiesto que existe un proceso de producción de sentido en el que práctica y discurso se imbrican, son simultáneas e inseparables.

Gran parte de los discursos curatoriales se articulan —en alguna medida - a partir de la teoría de la práctica, es decir, sustentan sus registros discursivos desde las experiencias narradas por otros curadores, críticos e historiadores, que instituyen un proyecto que refunda el sentido de una exposición. Esos discursos, convertidos en teoría, son mucho más que un soporte textual, son textualidades críticas que poseen un sello militante e identitario y que han sido claves para la delimitación de la representación en la historia de las exposiciones.

Medina se refiere a las prácticas artísticas y curatoriales ' como dispositivos fértiles para romper con los estereotipos, para reelaborar cartografías y para generar nuevos conceptos y lugares que interpelen a la ciudadanía del lado del Sur.

Es importante hacer la salvedad de que - aunque exista un parecido de familia importante- prácticas artís-
ticas, institucionales, culturales y curatoriales no son lo mismo, dado que responden a campos específicos. 
ARTíCULO

EVA NATALIA FERNÁNDEZ

Prácticas emergentes, espacio

curatorial y emplazamiento Sur
Entonces, en esta reflexión, intentaremos diferenciar los derroteros que tienen las prácticas, poniendo especial atención al ámbito curatorial, articuladas con un territorio transversal: el campo del arte y el espacio expositivo, que posibilitan distinguir dos tipos de prácticas curatoriales: ${ }^{2}$ una avalada y sostenida por el discurso oficial del campo curatorial institucionalizado; y otra, disidente, establecida por la oposición a la narrativa canónica - propuestas por el historiador del arte y curador Marcelo Pacheco— emplazadas en un espacio geopolítico: el Sur.

\section{CONDICIONES DE POSIBILIDAD DE UNA PRÁCTICA}

No existe una única definición de lo que es una "práctica". Sin embargo, las definiciones oscilan entre:

a. la acción concreta de un sujeto o grupo dentro de un contexto determinado; y

b. como experiencia, habilidad o ejercicio reiterado de una actividad.

Así como las prácticas parecen someterse a los imperativos del contexto, su ejecución más inmediata remite a la praxis y al desenvolvimiento adecuado dentro de un campo. Las prácticas emergen en un sistema, como corolario de proyectos comunes o individuales, detentando el conocimiento del área de trabajo y su estructura programática. En ese sentido, la noción de "emergencia" - que se vincula al texto Estética de la emergencia de Reinaldo Laddaga - se vuelve muy sugestiva para realizar una descripción que dé cuenta del dominio de la práctica, dice:

¿Qué es la emergencia? Lo que las ciencias de la complejidad llaman emergencia tiene lugar en sistemas de elementos que realizan acciones simples, que bien pueden estar gobernados en su interacción (o, mejor, intra-acción, para usar la expresión de Karen Barad) por leyes simples, pero que cuando se juntan en campos de actividad o impacto producen regularidades que ningún examen de ellos por separado hubiera permitido anticipar. (Laddaga, 2006: 287).

Retomando la idea de experiencia, una práctica emerge de un expertise, de la disciplina de una acción repetida que es dominada y que en colaboración con otros produce una regularidad que persiste. Entonces, lo que emerge es algo nuevo, es un proyecto construido desde una transición que se da en el "conocer y hacer".

2 En una primera instancia la propuesta es de Marcelo Pacheco, sin embargo, son muchos los teóricos, curadores, críticos e historiadores que realizan la misma distinción en relación al ejercicio de la práctica y que tiene que ver con una posición política e ideológica, cercana a una militancia desde el Sur como una región sin límites territoriales precisos. 
ARTíCULO

EVA NATALIA FERNÁNDEZ

Prácticas emergentes, espacio

curatorial y emplazamiento Sur
Cuando define práctica, Laddaga habla de proyectos en los que “[...] se trata de cultivar la potencialidad de una situación para organizarse de manera tal de producir persistencias" (Laddaga, 2006: 289). No sólo piensa en un resultado, sino que centra su atención en los procesos de organización y desorganización que, afirma, “[...] escapan a los actos individuales de los agentes que por medio de ella se integran y de la cual se re-apropian en el curso de una práctica” (Laddaga, 2006: 289).

Raymond Williams, en el capítulo "Dominante, residual y emergente" de su texto Literatura y Marxismo, también atiende la importancia de los procesos culturales como interacciones complejas y dinámicas que, de alguna manera, dan sentido a las prácticas posteriores producto de esos procesos. Williams (1988:145), entiende lo emergente como: "[...] en primer término, los nuevos significados y valores, nuevas prácticas, nuevas relaciones y tipos de relaciones que se crean continuamente". No obstante, eso que emerge no es esencialmente nuevo, el autor insiste en que es imposible distinguir qué elementos son efectivamente alternativos, cuáles son residuales y otros característicos de la cultura dominante. Al hablar de relaciones ya se da por sentado que intervienen elementos conjugados que transitarán hacia otra fase del proceso cultural.

Este es uno de los giros que interesa para la discusión: entender a ese proceso cultural que crea prácticas como una narrativa fundante de las persistencias y regularidades que orientarán los campos en los que éstas emerjan. Entonces, en esta coyuntura, vale la pena mencionar los derroteros o posibilidades que configuran el sentido de las prácticas.

Las prácticas curatoriales en América Latina están sostenidas por una tradición que, si bien se empata con la aparición del curador como figura central, ha tenido un auge considerable a partir de la proliferación de exposiciones y espacios dedicados al arte y por la aparición de programas educativos en torno a la labor curatorial.

La historiadora del arte Angélica González Vásquez le otorga especial atención a ese punto, dado que estas nuevas apuestas académicas y el floreciente brote de literatura y teoría alrededor de esa práctica "[...] ha hecho que se comience a hablar de "lo curatorial" en el sentido de un campo disciplinar: lo curatorial sería precisamente ese deslizamiento hacia una disciplina con sus propios objetos de reflexión, sistemas de referencias, cánones, metodologías, etc." (Vásquez, 2016: 142)

El campo curatorial, para Vásquez, está asociado con las actividades específicas que realiza un curador y con una práctica enmarcada en la búsqueda de articular un discurso con la obra que se expone. Ella lo denomina "la construcción de un argumento" que pondría en diálogo una exposición, un dispositivo espacial y una selección de objetos (Williams, 2016: 142).

Ahora bien, existe algo que progresivamente se repite en la literatura especializada en el análisis y la reflexión respecto a las prácticas curatoriales: el imperativo sobre ciertas capacidades - ¿innatas? - del curador. Sin un manejo de las técnicas, de las habilidades y la posesión de ese saber, para algunos teóricos no habría práctica; y esta exigencia parece 
ARTíCULO

EVA NATALIA FERNÁNDEZ

Prácticas emergentes, espacio

curatorial y emplazamiento Sur ser una demanda de orden global. Incluso, los curadores de renombre arguyen la imposibilidad de enseñar "cómo curar" una exposición, ya que esa práctica se adquiere sin escuela, es un ejercicio de formación curatorial en el mismo emplazamiento.

Este campo, al institucionalizarse como disciplina, abre la puerta a la reflexión crítica sobre "lo curatorial" y demuestra que la formación de curadores especializados se puede realizar desde la práctica y, también, desde la teoría. Se han incorporado nuevas demandas que requieren otros abordajes, del mismo modo que se ha producido una expansión del campo y del ejercicio mismo de la práctica. A lo largo de las últimas dos décadas han aparecido revistas, simposios y posgrados que dan cuenta de la importancia que se le ha otorgado a la curaduría mostrando un "[...] ascenso de la visibilidad del curador dentro del campo del arte contemporáneo" (O’Neill, 2012: 31).

En esta inflexión, habría que mencionar que la curaduría de una exposición no es una acción programática ni estática. Está claro que cada dispositivo de exposición tiene características singulares, pero sobre todo, el curador realiza un recorte de los objetos que tiene enfrente, toma decisiones.

En el texto "Las múltiples caras del curador", Jonathan Feldman reflexiona sobre esta idea a través de la figura de las funciones y de las tareas del curador como el creador de un metadiscurso. Expone:

De esta manera, la práctica curatorial estaría identificada, por un lado, con decidir sobre el recorte de obras que se exponen y, por otro lado, con una condición de reconocimiento de los trabajos de los artistas que - a su vez- sería al mismo tiempo una condición de producción de un nuevo discurso (el curatorial) (Feldman, 2012: 3).

Desde distintas perspectivas teóricas, si leemos la práctica curatorial como un proceso cultural que involucra elementos - un curador, un repertorio de obras y un espacio expositivo-y produce regularidades que persisten (Laddaga, 2006), estamos frente a un sujeto que transforma elementos preexistentes y residuales en otros emergentes (Williams, 2016). Y en la concreción de esa práctica nueva se efectuó una selección arbitrada por un curador. En esta suerte de desambiguación, sobre las funciones del curador, es donde reposa la necesidad de reflexión más profunda sobre el campo y sus alcances. Curadores de renombre, como Gerardo Mosquera, Mari Carmen Ramírez, Paul O'Neill, han dedicado libros y artículos que recuperan la labor de este sujeto que se ha visto en la necesidad de multiplicar sus funciones. En el texto "La emergencia del discurso curatorial desde el final de los años sesenta hasta el presente", se argumenta:

Esta ampliación de la noción del curador como un agente responsable de la estructura de conjunto de la exposición y de la narrativa, estableció un uso ahora ubicuo de la frase "curada por" (en el contexto de las invitaciones de las exposiciones, las notas de prensa y los catálogos). Como el 
ARTíCULO

EVA NATALIA FERNÁNDEZ

Prácticas emergentes, espacio

curatorial y emplazamiento Sur atributo normativo de todas las muestras, "curada por" articula un rol de autoría semiautónomo para el curador (O’Neill, 2012: 17).

Las caras del curador, atendiendo a sus múltiples posibilidades operativas son muchas y han mutado a través del tiempo. De comisario de sala, técnico y cuidador de obra hasta el de autor, lugar más reconocido en el mundo del arte contemporáneo y que está sostenido por una función enunciativa.

\section{LA EMERGENCIA DEL SUR EN EL CAMPO CURATORIAL}

A partir de la década de los ochentas, aproximadamente, este espacio Sur -imaginado o delimitado territorialmente-cobró fuerza, especialmente porque se vigorizó y expandió la labor de los curadores que tienen un discurso enunciado desde este sitio. Aparecieron figuras fundamentales en la escena latinoamericana como Gerardo Mosquera, Gustavo Buntinx, Cuauhtémoc Medina, Mari Carmen Ramírez y Marcelo Pacheco, por mencionar algunos. Esta presencia curatorial se convirtió en un acto enunciativo, en palabras de Justo Pastor Mellado (2001): en un "performance instituyente". Porque estos curadores fueron los que cimentaron, a través de sus discursos y sus prácticas, "el modo de hacer" curaduría, emplazados en un campo Sur y tratando de reformular los relatos verticales establecidos desde el norte. Un ejemplo de esto, se encuentra evidenciado en el artículo "Campos de batalla: historia del arte vs práctica curatorial" de Marcelo Pacheco (2001), donde el autor presenta el estado de la cuestión metodológico y epistémico de la acción curatorial latinoamericana y su intrínseco vínculo con la historia del arte. La propuesta de Pacheco parece dirigirse hacia cómo la práctica se convierte en un instrumento potencial para la renovación y la autonomía de discursos alternativos o marginalizados. Identifica dos derroteros importantes que pondrían a prueba el perfil político de los mismos curadores o críticos - que muchas veces fungen como tales- en el momento de la elaboración del aparato discursivo de las exposiciones. En su artículo describe las dos posibles rutas de un trabajo curatorial que reproduce significados - ajenos y agotados que legitiman los mismos mandatos de Europa y del Norte - frente a otra práctica que logra desligarse de las narrativas globalizadas, que desarticula y deconstruye los discursos, que trabaja desde los márgenes, que enuncia desde el marco y apuesta a la polisemia de sentido.

Cualquiera de las dos es para Pacheco una toma de posición. Sin embargo, esa práctica alternativa se convierte en “[...] campo de escritura, de edición, de re-inscripción, por fuera de los discursos auto referentes de la modernidad, y por fuera de la lógica pragmática y utilitaria del capitalismo tardío" (Pacheco, 2001: 4).

Pensamos que habría una responsabilidad crucial en el ejercicio de la práctica curatorial en América Latina en oposición a la de los países del norte, porque ese "hacer situado" abriría la puerta a una re-interpretación de las producciones culturales y artísticas 
ARTíCULO

EVA NATALIA FERNÁNDEZ

Prácticas emergentes, espacio

curatorial y emplazamiento Sur latinoamericanas y a la posibilidad de tejer nuevas relaciones que impliquen valores, saberes y poderes. Por qué no proponer también una "curaduría glocal", como una estrategia capaz de articular discursos globales con prácticas locales y viceversa. Práctica que Justo Pastor Mellado, llama "productor de infraestructuras", en oposición a un "curador de servicio". Esta distinción sostiene la ya expuesta por Pacheco, para Mellado, un productor de infraestructura es un curador que busca transformar un terreno que carece de un soporte cultural que le de libertad de acción en la escena local, y uno de servicio, refiere a la estrategia de edición de un guión curatorial asignado y avalado por la industria global de las exposiciones.

Este saber hacer curatorial, disidente, que proponen Pacheco y Mellado, es la forma de construcción de un lenguaje que utiliza como soporte los elementos del espacio expositivo subvirtiendo los rezagos coloniales heredados de la historia del arte. Es una narración alternativa que se desplaza reconfigurando el sentido y la legitimidad de los nuevos discursos. Y Pastor Mellado agregaría, sin dejar de lado el fortalecimiento de una infraestructura cultural articulada a una historia que sustente o apele a una re-localización efectiva del arte latinoamericano. Sostiene que:

El curador de servicio trabaja para fortalecer las redes de consistencia de las corporaciones, en el terreno específico en el que se juega la rentabilidad simbólica de la marca. Sobre este fondo, admito que la distinción entre curador de servicio y curador/productor de infraestructura debe satisfacer un imperativo dogmático, destinado a separar abruptamente estilos y estrategias de trabajo (Mellado, 2001: s.p.).

En el ámbito práctico de la institución, las obras y los objetos de arte son los elementos que configuran una gestión expositiva. En este sentido, la idea de Pacheco de que el acto de exponer tendría que entenderse como un acto discursivo, refuerza el poder de la práctica curatorial como un dispositivo que interviene muchos espacios -incluso el simbólico- haciendo pública una clasificación de elementos ordenados que produce significado. En esta suerte de sesgo, del encuadre de lo que se expone y se muestra, se advierte una intención y una mirada, así como el intersticio para sostener una posición comprometida y política.

Paul O'Neill, en uno de sus libros, explica que recién desde la década de los noventa fue posible rastrear textos y publicaciones especializadas en "la historia de las exposiciones", "las innovaciones curatoriales" y "la institucionalización de la función curatorial". Estas categorías se consolidaron al interior de los debates de la época y se materializaron en una nueva industria editorial operada por curadores jóvenes con un epicentro común: el arte contemporáneo y su promoción como plataforma de investigación. Porque la relación entre el acto curatorial, los modos en los que se traspasan las fronteras de la producción artística y la responsabilidad de la autoría y su mediación, abren un campo totalmente nuevo que desmitifica al curador como un simple intermediario de saberes. 
ARTíCULO

EVA NATALIA FERNÁNDEZ

Prácticas emergentes, espacio

curatorial y emplazamiento Sur
El curador Bruce Altshuler considera que el boom de las exposiciones — signado en gran medida por la irrupción de la figura del curador independiente desde los años sesenta, como organizador de muestras de arte contemporáneo fuera del museo-fue un acontecimiento decisivo para desmontar modelos - como la vanguardia histórica o la historia del modernismo-, ya que instalaba debates críticos al interior de estos dispositivos alternativos. Por ello, las exposiciones temporales fueron un parteaguas que inauguraría una forma viable de organización, de orden, de lectura y de sentido de un acotado repertorio de objetos. O 'Neill las llama "cápsulas del tiempo", porque son exposiciones breves y efímeras, lo difícil fue documentar y realizar un registro fiel de lo que aconteció en cada una de ellas.

Bien, el creciente número de exposiciones de naturaleza fugaz, en espacios experimentales que no siempre respondían al cubo blanco, se multiplicó permitiendo expandir las posibilidades de sentido y de enunciación. Muchos curadores independientes comenzaron a realizar muestras en espacios alternativos, los casos más ilustrativos son los de Germano Celant, Walter Hoops, Pontus Hultén, Seth Siegelaub y Harald Szeemann. Podríamos sugerir, que esta forma de emplazamiento de las exposiciones logró convertirlas en dispositivos críticos que transformaron la práctica expositiva, no sólo por la actualización metodológica y epistémica de su ejecución, sino por su inagotable gama de posibilidades como una plataforma abierta a la significación.

Entonces, la multiplicación de exposiciones en todo el globo reactualizó la forma de curar, de exhibir, de concebir y de discutir los temas y el modo de proyectarlos.

Las exposiciones realizadas en el Sur, también tienen una historia y un sello político disonante. Se pueden traducir como el esfuerzo compartido de curadores, críticos y artistas por detentar un lugar de disidencia dentro del incontenible proceso de globalización que se extendería a todas las esferas sociales. La enunciación desde el Sur es el reclamo de una "territorialidad imaginada" ${ }^{3}$ que, si bien está sometida a condicionamientos institucionales, regidos por viejos paradigmas, intenta re-orientar la práctica curatorial estableciendo otros puntos de vista y nuevas identidades.

A propósito de los curadores latinoamericanos que apuestan por una práctica retractora de los argumentos gestados en el otro hemisferio, Cuauhtémoc Medina describe lo que representa el "Sur" en su trabajo, dice:

El término Sur resultaba ampliamente cargado de una politización de la geografía: era más un llamado a cambiar de orientación en las referencias y estudios que un concepto a ser transmitido como realidad empíricamente verificable. Era el marcador de un punto de vista (Medina, 2015: 112). Como un guiño al texto Comunidades imaginadas de Benedict Anderson (1983). 
ARTíCULO

EVA NATALIA FERNÁNDEZ

Prácticas emergentes, espacio

curatorial y emplazamiento Sur
En el mismo tenor, Gustavo Buntinx se refiere a esta restitución del proceso cultural "[...] Surgida por fuera de las lógicas de la mirada metropolitana" como "[...] el empoderamiento de lo local" (Buntinx, 2005). Piensa que una demarcación latinoamericana legítima sólo es posible si se cumplen algunas simultaneidades como una diversificación del mercado, una institución de arte autónoma y la incorporación de los artistas como agentes de cambio en el proceso democrático de la región.

Este llamado a trazar nuevos mapas es una apelación a la emancipación fehaciente de la práctica del Sur, es establecer un sitio para reescribir las historias y desmantelar verticalidades, es agudizar la mirada e identificar las narrativas dominantes para no caer en ellas. Como afirma Medina, "[...] es la postulación de una comunidad generada en gran medida por las consecuencias de cuestionar la exclusión y los estereotipos de consumo del exotismo" y "[...] se propone todo el tiempo habitar una práctica cultural atravesada por el dilema ético de la traducción cultural" (Medina, 2015: 112).

Este escenario, deseable, de renovación de las prácticas curatoriales ha generado opiniones dispares dentro del circuito curatorial del arte. Mari Carmen Ramírez, por ejemplo, en "Mediación identitaria: los curadores de arte y las políticas de representación cultural", sin referirse específicamente a la práctica curatorial desde el Sur, sostiene que es empíricamente imposible. Ella identifica dos escollos en el camino: a) el curador no actúa como un agente activo de promoción de las producciones artísticas periféricas; y b) generalmente, los curadores no pueden inaugurar una práctica crítica sin caer en el "consumo de falsas diferencias" (Ramírez, 2008: 23).

Ramírez, como curadora especializada que ha trabajado durante mucho tiempo en Estados Unidos, reconoce que los curadores latinoamericanos - más cercanos al rol de "brokers culturales", agentes de intercambio cultural - ganan visibilidad en los circuitos artísticos a partir de aliarse con los grupos de poder y asumir sus intereses, o, de realizar una práctica guiada por el mercado cultural de las identidades. Parece, que las buenas intenciones y la consolidación de una práctica curatorial que evada la lógica del capitalismo de consumo se articula — como lo describe la autora - con las mediaciones de un "[...] márketing de la apariencia, ya sea de la "diferencia" o bien de la particularidad" (Ramírez, 2008: 13). Lejos de buscar aliarse con la voz de uno o de otro, sobre las posibilidades de marcar una diferencia en el campo curatorial del Sur o latinoamericano, parece necesario poner sobre la mesa que para algunos curadores e historiadores - con una trayectoria importante en el círculo curatorial- no es tan fácil, desde el ejercicio de la práctica, detentar una posición comprometida con la región.

\section{PRÁCTICAS EN EL CAMPO DEL ARTE}

Prácticas, campo y arte latinoamericano, son tres registros analíticos que deberían ser parte de una agenda que apele a signar una posición geopolítica que hable de resistencia y de re-localización. Así lo pensamos algunos. 
ARTíCULO

EVA NATALIA FERNÁNDEZ

Prácticas emergentes, espacio

curatorial y emplazamiento Sur
El arte latinoamericano, como marco epistémico para el desarrollo de las exposiciones y la práctica curatorial, artística e institucional, parece ser un campo que sigue fungiendo como blanco de diversos debates. Siendo justamente eso, un marco, el tránsito que ha tenido la historia, o las historias, del arte desde mediados del siglo XX aporta datos interesantes que se articulan a coyunturas geopolíticas, culturales, económicas y artísticas.

Si bien en esta investigación no se realiza un recorrido exhaustivo, de tipo historiográfico, debemos advertir que su impronta - la del arte latinoamericano- ha dejado huellas insoslayables para pensarnos, hoy, dentro de los campos del arte y la cultura.

Diana Wechsler, curadora e historiadora del arte argentina, atendiendo a su experiencia curatorial en distintos puntos del globo, en el artículo "¿De qué hablamos cuando decimos Arte Latinoamericano? Exposiciones y perspectivas críticas contemporáneas", cree que son las exposiciones - bienales y colecciones de arte latinoamericano - las que organizan y llenan de sentido las nociones que se construyen dentro de los centros y campos de arte. Cual una agenda estipulada para repensar lo latinoamericano en el seno de su producción, Wechsler piensa que allí es donde se forja el pensamiento crítico sobre el arte contemporáneo. En estos encuentros circulan las "nociones" que posteriormente resonarán en las producciones artísticas latinoamericanas como la identidad, lo local, lo regional y la otredad. Que son como una suerte de postead en blanco, derivas construidas para ser llenadas de sentido según las políticas del poder en mando, así lo describe la autora, hace la salvedad que "[...] se inscriben dentro de la lógica del nuevo orden mundial" (Wechsler, 2012: 7).

Entonces, la circulación de esas nociones proyectadas en los campos artísticos y curatoriales las transforma en elementos clave para pensar los procesos culturales latinoamericanos, y del Sur, con el afán de entender cómo será esta representación a nivel curatorial e institucional.

La noción de "campo" de Pierre Bourdieu, desarrollada en extenso en distintos libros del autor, arroja luz para vincular la categoría a los espacios curatoriales, en donde se establecen pautas importantes que instituyen el sentido. Campo como un modo de pensar a partir de relaciones. En un sistema de posiciones, los actores están definidos por su capacidad relacional y no por ser elementos autónomos, esas relaciones tienen una estructura temporal y una función precisa dentro del campo. Los movimientos que realizan los actores tienen que ver con el interés y con el valor que se otorga a determinados bienes culturales y simbólicos. Y cuando hablamos de bienes nos estamos refiriendo a campos de producción. En El sentido social del gusto, Bourdieu da pautas metodológicas para pensar los campos sociales como pequeños eslabones de un sistema de relaciones en el que se producen, circulan y se demandan determinados bienes simbólicos.

Estas lógicas de funcionamiento, permiten pensar un campo curatorial como un sistema de relaciones jugadas por actores e instituciones que tienen diferentes roles dentro de él, algunos con más autoridad y otros con menos, que sostienen y definen las condiciones para la pertenencia al grupo, para el establecimiento de reglas, para las condiciones de acceso, y, sobre todo, para la selección de quienes ocupan una posición de poder. Entonces, los 
ARTíCULO

EVA NATALIA FERNÁNDEZ

Prácticas emergentes, espacio

curatorial y emplazamiento Sur agentes que dominan las ideas son lo que legitiman el valor cultural, artístico e intelectual de los bienes simbólicos estableciendo una jerarquización. En este sentido, la definición propuesta por el sociólogo francés se puede atribuir, particularmente, a la escena del arte latinoamericano -en este caso a partir de un diálogo nutrido entre los curadores, la crítica y las instituciones- que está ligado al complejo entramado de producción y circulación que impone la red del mercado del arte global.

Son diversos los autores que recuperan la voz de Pierre Bourdieu, sin embargo, Néstor García Canclini señala una experiencia distinta del campo. En la conferencia titulada "Geopolítica del arte y estéticas interculturales", Gracía-Canclini analiza distintas condiciones actuales que anulan los proyectos sociológicos o estéticos que configuran los sentidos sociales. Uno de ellos es el "desdibujamiento de los campos artísticos". No nos adelantamos a esta cuestión. El autor, primero, describe la formación de los campos de producción del arte y muestra cómo se han modificado los actores que dictan e imponen las reglas dentro de los mismos. Ya no es más el Estado o la Iglesia los que estipulan las reglas del juego, sino - y en sintonía con la teoría de Pierre Bourdieu- "[...] la consagración de las obras y de los artistas, y por tanto la producción de una creencia social en su valor, pasó a ser conferida por actores especializados: museos, editores, críticos, lectores y espectadores" (García Canclini, 2008: 2).

La configuración de los campos y sus prácticas define en qué arena se debatirán las políticas culturales que someten al arte a un régimen estrictamente institucional. Entonces, Canclini se pregunta:

¿Qué es lo que constituye a un campo? Dos elementos: la existencia de un capital común y la lucha por su apropiación. A lo largo de la historia, el campo científico o el artístico han acumulado un capital (de conocimiento, habilidades, creencias, etcétera) respecto del cual actúan dos posiciones: la de quienes detentan el capital y la de quienes aspiran a poseerlo. Un campo existe en la medida en que uno no logra comprender una obra (un libro de economía, una escultura) sin conocer la historia del campo de producción de la obra (García Canclini, 1990: 11).

Si desde el siglo pasado, como sostiene Andrea Giunta, "[...] las exposiciones organizadas desde fines de los años cincuenta hasta el presente y en los libros editados sobre arte latinoamericano, puede rastrearse un conjunto de ideas rectoras, que siguen, en gran parte, pautando las historias más recientes" (Giunta, 1996: 2), no se debe subestimar el poder de control, del sentido social y cultural, que circula y se reconoce al interior de un campo.

La afirmación de Giunta podría habilitar una reflexión anunciada por Canclini: el desdibujamiento de los campos artísticos. El autor dice: "[...] el trabajo crítico se ve en la necesidad de trascender los "círculos de reconocimiento" (García-Canclini, 1990). Es decir, trabajar por una práctica autónoma que no se encuentre signada por las lógicas del mercado. Marcelo Pacheco propone, en el mismo tenor, pensar las prácticas como narraciones 
ARTí́CULO

EVA NATALIA FERNÁNDEZ

Prácticas emergentes, espacio

curatorial y emplazamiento Sur -agregaríamos performativas - como si fueran discursos que describen la urgencia de abogar por espacios de debate y poder. Pacheco apela a la capacidad relacional de las prácticas como una zona privilegiada de acción, objeta que "[...] lo interesante es que la práctica curatorial es, en sí misma, un campo de escritura que trabaja sobre los agujeros, que propone umbrales, que teje pliegues y repliegues en una trama incesante. No fija objetos, sino que se desliza incierta abriendo campos de relaciones (Pacheco, 2001: 4).

\section{REFLEXIONES FINALES}

Las prácticas curatoriales en América Latina deben concebirse como un instrumento que, alineado a una localización desde el Sur, en congruencia con Pacheco y Medina, se convierta en un lugar de enunciación.

Cuando Cuauhtémoc Medina dice "Curando desde el Sur" “[...] remite a una práctica que se configura cuando uno se ubica - o uno descubre que está- en una dialéctica de debates históricos a largo plazo; cuando uno se asume como parte de una genealogía crítica que negocia, desespera o redefine la historia del Sur como una geografía disidente del arte moderno y contemporáneo" (Medina, 2015: 112).

Las prácticas, como se expuso en un inicio, son modos de "saber hacer"; son ejecuciones, muchas veces programáticas, que remiten a la experiencia y a las capacidades de un sujeto. Si se asume que ellas emergen en un campo curatorial, las posibilidades de desplazamiento y movilidad para desmontar algunas inercias y abrir nuevos campos de relaciones es potencial. Porque cual mapas en los que se dibuja el sentido, se yuxtaponen valores, intereses, poderes, localización y gusto. Son proyectos que abren un diálogo para constituir otras formas de conocimiento crítico.

En este sentido las exposiciones y presentaciones de arte latinoamericano son dispositivos articulados al discurso curatorial, son iniciativas que responden a una geografía y que, entendidas como marco epistémico, pueden transitar hacia otras formas de acción que interpelen los procesos de diferencias culturales tan presentes en América Latina. Así como sostiene Justo Pastor Mellado, los curadores anclados al espacio Sur deberían ser productores de una infraestructura que decodifique y ampare las producciones artísticas latinoamericanas. La apuesta, y el reto, se encuentra en reconfigurar críticamente el campo curatorial Sur a partir de una práctica alternativa, de la que habla Pacheco, que gestione un proceso de edición de los discursos y que sostenga esa posición que busca desacoplar las representaciones impuestas por el norte renovando las lógicas.

El último punto, quizá el más álgido, propondría repensar con conciencia si es "el Sur" la categoría que guiará la práctica curatorial contemporánea. Porque un relato enunciado desde esa posición localizada también es transformable en un discurso de exclusión que limita las posibilidades de sentido y las propuestas alternativas que buscan contar otras historias. I 
ARTí́CULO

EVA NATALIA FERNÁNDEZ

Prácticas emergentes, espacio

curatorial y emplazamiento Sur

\section{REFERENCIAS}

Buntinx, G. (2005). “El empoderamiento de lo local” [Ponencia]. En Foro Circuitos latinoamericanos/circuitos internacionales: interacción, roles y perspectivas, Feria de Arte de Buenos Aires. Disponible en: https://www.micromuseo.org.pe/lecturas/gbuntinx.html

Feldman, J. (2012). "Las múltiples caras del curador" [Ponencia]. En Tercera jornada de estudiantes y graduados en Crítica de Arte, IUNA, Buenos Aires. Disponible en: https://www.academia.edu/14877203/Las_múltiples_caras_del_curador

García-Canclini, N. (2008). "Geopolíticas del arte y estéticas interculturales" [Conferencia], Universidad de Miami. Disponible en: http://nestorgarciacanclini.net/index.php/ estetica-y-antropologia/78-conferencia-qgeopolitica-y-esteticas-interculturalesq

García-Canclini, N. (1990). "Introducción”. En P. Bourdieu Sociología y cultura. México: Editorial Grijalbo, pp. 9-50.

Giunta, A., (1996), “América Latina en disputa. Apuntes para una historiografía del arte latinoamericano" [Ponencia]. En Investigaciones Estéticas and Rockefeller Foundation, Oaxaca.

González-Vásquez, A. (2016). “Un giro educativo: de la práctica de la curaduría a lo curatorial”. En Errata, núm. 16, pp. 140-159. Disponible en:

http://www.revistaerrata.gov.co/contenido/ un-giro-educativo-de-la-practica-de-la-curaduria-lo-curatorial

Laddaga, R. (2006). Estética de la emergencia: la formación de otra cultura de las artes. Buenos Aires: Adriana Hidalgo Editora.

Medina, C. (2015). "Curando desde el Sur una comedia en cuatro actos". En Errata, núm. 14, pp. 108-123. Disponible en:

https://www.revistaerrata.gov.co/contenido/ curando-desde-el-Sur-una-comedia-en-cuatro-actos

Mosquera, G. (1994). "Algunos problemas del comisariado transcultural”. En Global Vision. Toward a New Internationalims in the Visual Arts. London: Kala Press/INIVA.

O'Neill, P. (2012). "The emergence of curatorial discourse from the late 1960 to the present". En The culture of curating and the curating of culture. E.U.: The MIT Press.

Pacheco, M. (2001. “Campos de batalla...Historia del arte vs. práctica curatorial”. En Micromuseo al fondo hay sitio. Museo Antropológico y de Arte Contemporáneo de Guayaquil. Disponible en: https://www.micromuseo.org.pe/lecturas/mpacheco.html

Mellado, P. (2001). "Apuntes para una delimitación de la noción de curador como producción de infraestructura". Micromuseo al fondo hay sitio. Museo Antropológico y de Arte Contemporáneo de Guayaquil. Disponible en: https://www.micromuseo.org.pe/lecturas/ jpastor.html

Ramírez, M. (2008), “Mediación identitaria: los curadores de arte y las políticas de representación cultural". En Revista Ramona, núm. 86, pp. 9-23. Disponible en: http://70.32.114.117/gsdl/ collect/revista/index/assoc/HASH917c/8doff48d.dir/r86_ognota.pdf 
ARTí́CULo

EVA NATALIA FERNÁNDEZ

Prácticas emergentes, espacio

curatorial y emplazamiento Sur
Williams, R. (1988). Marxismo y literatura. Barcelona: Ediciones Península.

Wechsler, D. (2012). “¿De qué hablamos cuando decimos Arte Latinoamericano? Exposiciones y perspectivas críticas contemporáneas". En Caiana. Revista electrónica de Historia del Arte y Cultura Visual del centro argentino de investigadores del arte (CAIA), núm. 1, pp. 1-9. Disponible en: http://caiana.caia.org.ar/resources/uploads/1-pdf/DianaWechsler.pdf 